\title{
A Least Squares Procedure for the Wave Equation
}

\author{
By Alfred Carasso *
}

\begin{abstract}
We develop and analyze a least squares procedure for approximating the homogeneous Dirichlet problem for the wave equation in a bounded domain $\Omega$ in $R^{N}$. This procedure is based on the pure implicit scheme for time differencing. Surprisingly, it is the normal derivative of $u$ rather than $u$ itself which must be included in the boundary functional. This normal derivative is an unknown quantity. We show that it may be set equal to zero while retaining the $O(k)$ accuracy of the pure implicit scheme. The penalty is that one must use smoother trial functions to obtain this accuracy.
\end{abstract}

1. Introduction. In a recent paper [4], Bramble and Thomée describe least squares methods for the heat equation in a bounded domain $\Omega$ in $R^{N}$, under Dirichlet boundary conditions. The importance of such a procedure, in the context of the numerical computation of time dependent problems by variational methods, is clear. Unlike the usual Galerkin methods, the trial functions need not satisfy the boundary conditions, and hence one can handle problems in a general domain. Moreover, the ideas developed in [4] would seem to have application to other evolution equations. In the present note, we develop the corresponding theory for the wave equation. We hope to consider other time dependent problems in a later report. Since the discussion in [4] is very transparent, we have adhered closely to the notation and organization of [4]. For the sake of brevity, we will only consider a least squares procedure based on the "pure implicit" scheme. However, the discussion is easily extended to the Crank-Nicolson scheme. It would be interesting to construct least squares methods based on explicit schemes, since, for hyperbolic problems, the requisite stability conditions are tolerable.

2. The Analytic Problem. Let $\Omega$ be a bounded domain in $R^{N}$ with a smooth boundary $\partial \Omega$. We seek to approximate the solution of the mixed problem

$$
\begin{aligned}
u_{t t} & =\Delta u, & & x \in \Omega, \quad t>0, \\
u(x, 0) & =f(x), & & x \in \Omega, \\
u_{t}(x, 0) & =g(x), & & x \in \Omega, \\
u & =0, & & x \in \partial \Omega, \quad t \geqslant 0 .
\end{aligned}
$$

It is convenient to make use of the spaces $\dot{H}^{s}, s \geqslant 0$, introduced in [4]. Let $\left\{\lambda_{m}\right\}_{m=1}^{\infty}$ be the (positive) eigenvalues of the negative Laplacian in $\Omega$, with zero Dirichlet data on $\partial \Omega$, and let $\left\{\varphi_{m}\right\}_{m=1}^{\infty}$ be the corresponding orthonormal sequence of eigenfunctions. For a given $v \in L^{2}(\Omega)$, let $\left\{\beta_{m}\right\}_{m=1}^{\infty}$ be the sequence of Fourier coefficients of $v$ relative to the $\left\{\varphi_{m}\right\}$. For $s \geqslant 0$, the Hilbert space $\dot{H}^{s}$ is defined to be the subspace

Received June 1, 1973; revised August 27, 1973.

AMS (MOS) subject classifications (1970). Primary 65M15. 80302 .

* Research supported by the National Center for Atmospheric Research, Boulder, Colorado 
of $L^{2}(\Omega)$ consisting of all $v$ 's for which the norm

$$
\|v\|_{s}=\left(\sum_{m=1}^{\infty} \lambda_{m}^{s}\left|\beta_{m}\right|^{2}\right)^{1 / 2}
$$

is finite. Since $\partial \Omega$ is assumed well-behaved, it follows that if $s \geqslant 1$ and $v \in \dot{H}^{s}$, then $v=0$ on $\partial \Omega . \dot{H}^{\infty}=\bigcap_{s>0} \dot{H}^{s}$ is dense in every $\dot{H}^{s}$, and if $s$ is a nonnegative integer, and $v \in \dot{H}^{\infty}$, then the $s$-norm (2.2) is equivalent to the usual Sobolev norm, $\|v\|_{H^{s}}$.

Expanding in the eigenfunctions of $\Delta$, in (2.1), one immediately obtains the following results concerning the well-posedness of problem (2.1).

THEOREM 2.1. Let $l \geqslant 0$. For each $f(x) \in \dot{H}^{l+1}, g(x) \in \dot{H}^{l}$, problem (2.1) has a unique solution, and the following holds:

$$
\left\{\|u(\cdot, t)\|_{l+1}^{2}+\left\|u_{t}(\cdot, t)\right\|_{l}^{2}\right\}^{1 / 2}=\left\{\|f\|_{l+1}^{2}+\|g\|_{l}^{2}\right\}^{1 / 2} .
$$

As indicated by Theorem 2.1, we may as well consider the first order system equivalent to (2.1). Let $G$ be the $2 \times 2$ matrix

$$
G=\left[\begin{array}{ll}
0 & I \\
\Delta & 0
\end{array}\right]
$$

and let $U(x, t)$ be the 2 component vector

$$
U(x, t)=[u(x, t), v(x, t)]^{T} .
$$

Putting $v(x, t)=u_{t}(x, t)$ in $(2.1)$, we obtain the equivalent problem

$$
U_{t}=G U, \quad U(x, 0)=[f(x), g(x)]^{T} .
$$

Defining the norm $\|U(t)\|_{s}$ by

$$
\|U(t)\|_{s}=\left\{\|u(\cdot, t)\|_{s+1}^{\|^{2}}+\|v(\cdot, t)\|_{s}^{2}\right\}^{1 / 2}, \quad s \geqslant 0,
$$

(2.3) becomes

$$
\|U(t)\|_{s}=\|U(0)\|_{s}, \quad s \geqslant 0, t>0 .
$$

If $s$ is a nonnegative integer, then by the equivalence of norms, we have

$$
\|U(t)\|_{H^{s}} \leqslant C_{s}\|U(0)\|_{H^{s}}, \quad t>0,
$$

for some positive constant $C_{s}$ depending only on $s$.

With $\lambda_{m}, \varphi_{m}$ the characteristic pairs of $-\Delta$ as above, the characteristic pairs of $G$ are given by

$$
\mu_{ \pm m}= \pm i \sqrt{\lambda_{m}}, \quad \Phi_{ \pm m}=\left[\varphi_{m}, \mu_{ \pm m} \varphi_{m}\right]^{T},
$$

and the $\Phi_{m}$ 's constitute an orthogonal basis in the Hilbert space $\dot{H}^{s+1} \times \dot{H}^{s}$ for each $s \geq 0$. If $\left\{\alpha_{m}\right\}_{m=-\infty}^{\infty}$ is the sequence of Fourier coefficients of the vector $V$ relative to the $\Phi_{m}$ 's, we note that the $s$-norm of $V$ defined by (2.7) is also given by

$$
\|V\|_{s}^{2}=2 \sum_{m=-\infty}^{\infty}\left|\mu_{m}\right|^{2 s+2}\left|\alpha_{m}\right|^{2} .
$$


3. The Semi-Discrete Problem. Let $k>0$ be a small increment in the time variable. Leaving the space variables continuous, we discretize the time in (2.6), using the pure implicit scheme, to obtain

$$
\begin{aligned}
(I-k G) W^{n+1} & =W^{n}, \quad n=0,1,2, \ldots, \\
W^{0} & =[f, g]^{T} .
\end{aligned}
$$

Expanding in the eigenvectors of $G$, it is easily seen that one can uniquely solve (3.1) for each $n$. For $t=n k$, let $E_{k}(t)$ denote the solution operator at time $t$ associated with the initial-value problem (3.1), (3.2). We then have

Theorem 3.1. Let $s \geqslant 0$ and let $W^{0} \in \dot{H}^{s+1} \times \dot{H}^{s}$. Then $E_{k}(t) W^{0} \in \dot{H}^{s+1} \times \dot{H}^{s}$ and

$$
\left\|E_{k}(t) W^{0}\right\|_{s} \leqslant\left\|W^{0}\right\|_{s} .
$$

Proof. Put $W^{0}=\sum_{m} \alpha_{m} \Phi_{m}$. Then,

$$
E_{k}(t) W^{0}=\sum_{m} \frac{1}{\left(1-k \mu_{m}\right)^{n}} \alpha_{m} \Phi_{m}
$$

Hence,

$$
\left\|E_{k}(t) W^{0}\right\|_{s}^{2}=2 \sum_{m}\left|\mu_{m}\right|^{2 s+2}\left|\alpha_{m}\right|^{2} /\left|1-k \mu_{m}\right|^{2 n} \leqslant 2 \sum_{m}\left|\mu_{m}\right|^{2 s+2}\left|\alpha_{m}\right|^{2}=\left\|W^{0}\right\|_{s}^{2} .
$$

We also have

THEOREM 3.2. Let $E(t)$ be the solution operator at time $t$ associated with the analytic problem (2.6). Then, for all $0 \leqslant t=n k \leqslant T$ and all $0 \leqslant s \leqslant 2$,

$$
\left\|E_{k}(t) W^{0}-E(t) W^{0}\right\|_{0} \leqslant \operatorname{Max}(2,2 T) k^{s / 2}\left\|W^{0}\right\|_{s} .
$$

Proof. To begin with, we note that with $\sigma$ real

$$
\left|1 /(1-i k \sigma)-e^{i k \sigma}\right| \leqslant 2 k^{2} \sigma^{2} .
$$

Since,

$$
\frac{1}{(1-i k \sigma)^{n}}-e^{i n k \sigma}=\left\{\frac{1}{(1-i k \sigma)}-e^{i k \sigma}\right\} \sum_{j=0}^{n-1} \frac{1}{(1-i k \sigma)^{j}} \cdot e^{-(n-1-j) i k \sigma}
$$

we have

$$
\left|\frac{1}{(1-i k \sigma)^{n}}-e^{i n k \sigma}\right| \leqslant 2 k^{2} \sigma^{2} \sum_{j=0}^{n-1} \frac{1}{|1-i k \sigma|^{j}} \leqslant 2 n k^{2} \sigma^{2} \leqslant 2 T k \sigma^{2} .
$$

Putting $i \sigma=\mu_{m}$ in (3.9), we see that, for $s=2$,

$$
\left|1 /\left(1-k \mu_{m}\right)^{n}-e^{\mu_{m} t}\right| \leqslant 2 T\left(k\left|\mu_{m}\right|^{2}\right)^{s / 2} \leqslant \operatorname{Max}(2,2 T)\left(k\left|\mu_{m}\right|^{2}\right)^{s / 2} .
$$

The last inequality is also valid when $s=0$. Hence, it is valid for $0 \leqslant s \leqslant 2$. Now, with $W^{0}=\sum_{m} \alpha_{m} \Phi_{m}$, we have $E(t) W^{0}=\sum \alpha_{m} e^{\mu_{m} t} \Phi_{m}$, while $E_{k}(t) W^{0}$ is given by (3.4). Therefore,

$$
\left\|E(t) W^{0}-E_{k}(t) W^{0}\right\|_{0}^{2}=2 \sum_{m}\left|1 /\left(1-k \mu_{m}\right)^{n}-e^{\mu_{m} t}\right|^{2}\left|\mu_{m}\right|^{2}\left|\alpha_{m}\right|^{2},
$$


and from (3.10), for $0 \leqslant s \leqslant 2$,

$$
\left\|E(t) W^{0}-E_{k}(t) W^{0}\right\|_{0} \leqslant \max (2,2 T) k^{s / 2} \cdot\left\{2 \sum_{m}\left|\mu_{m}\right|^{2 s+2}\left|\alpha_{m}\right|^{2}\right\}^{1 / 2} .
$$

This proves the theorem.

4. A Priori Estimates. Equations (3.1), (3.2) define a convergent "method of lines" for the approximate solution of (2.6). To actually implement this method, one must be able to approximately solve (3.1) at each time step. We shall construct a least squares procedure for this latter problem and eventually obtain a fully-discrete scheme for (2.6). As is well known, the stability of the semi-discrete scheme (3.1), (3.2) depends entirely on the dissipative character of the operator $G$ when applied to smooth functions satisfying the boundary conditions. In fact, backward time differencing is a classical device in proving the Hille-Yosida theorem [5, p. 479]. With certain finite difference analogs for the Laplacian, and with the usual Galerkin methods using trial functions satisfying the boundary conditions, this semiboundedness of $G$ is preserved. Hence, the resulting fully-discrete scheme is automatically stable. In the present methods, the trial functions do not satisfy the boundary conditions. It turns out to be an intriguing game to decide how to formulate the fully-discrete scheme so as to obtain stability. The construction hinges on certain a priori estimates which we develop in the present section.

We will be dealing with arbitrary elements of the Sobolev spaces $H^{s}(\Omega)$, s a nonnegative integer. Such functions will not usually belong to $\dot{H}^{s}$. Accordingly, we use the notation $(u, v)_{H^{s}},\|u\|_{H^{s}}$, for scalar products and norms in $H^{s}$. For two component vectors $U=[u, v]^{T}$, we define

$$
\|U\|_{H^{s}}=\left\{\|u\|_{H^{s+1}}^{2}+\|v\|_{H^{s}}^{2}\right\}^{1 / 2} .
$$

We will need the following trace theorem. See [1, p. 38] for the proof.

Lemma 4.1. Let $u \in H^{1}(\Omega)$ and let $\epsilon>0$. Then there exists a constant $\gamma$, independent of $u$ and $\epsilon$ and depending only on $\Omega$, such that

$$
\int_{\partial \Omega} u^{2} d s \leqslant \gamma\left\{\epsilon\|u\|_{H^{1}}^{2}+\frac{1}{\epsilon}\|u\|_{H^{0}}^{2}\right\} .
$$

Let $D(v, w)$ denote the Dirichlet integral

$$
D(v, w)=\int_{\Omega} \sum_{j=1}^{N} \frac{\partial v}{\partial x_{j}} \frac{\partial w}{\partial x_{j}} d x
$$

If $\nu$ is the exterior normal to $\partial \Omega$ and if $v$ and $w$ are smooth functions, we have by Green's formula

$$
\int_{\Omega} v \Delta w d x=\int_{\partial \Omega} v \frac{\partial w}{\partial \nu} d s-D(v, w)
$$

Let $L_{k}$ denote the operator $I-k G$ of Section 3. The following lemma is the basis of our procedure.

LEMMA 4.2. Let $U=[u, v]^{T}$ be an arbitrary element in $H^{2}(\Omega) \times H^{1}(\Omega)$. Then,

$$
(1-k)\|U\|_{H^{0}}^{2} \leqslant\left\|L_{k} U\right\|_{H^{0}}^{2}+\frac{1}{k^{2}} \int_{\partial \Omega} v^{2} d s+k^{4} \int_{\partial \Omega}\left|\frac{\partial u}{\partial v}\right|^{2} d s .
$$


Proof. We have

$$
L_{k} U \equiv U-k G U=[u-k v, v-k \Delta u]^{T}
$$

so that

$$
\begin{aligned}
\left\|L_{k} U\right\|_{H^{0}}^{2} & =(u-k v, u-k v)_{H^{1}}+(v-k \Delta u, v-k \Delta u)_{H^{0}} \\
& =\|u\|_{H^{1}}^{2}-2 k(u, v)_{H^{1}}-2 k(v, \Delta u)_{H^{0}}+\|v\|_{H^{0}}^{2}+k^{2}\|v\|_{H^{1}}^{2}+k^{2}\|\Delta u\|_{H^{0}}^{2} .
\end{aligned}
$$

Hence,

$$
\begin{aligned}
\|U\|_{H^{0}}^{2}-\left\|L_{k} U\right\|_{H^{0}}^{2} & =2 k(u, v)_{H^{1}}-k^{2}\|v\|_{H^{1}}^{2}+2 k(v, \Delta u)_{H^{0}}-k^{2}\|\Delta u\|_{H^{0}}^{2} \\
& \leqslant 2 k(u, v)_{H^{1}}+2 k(v, \Delta u)_{H^{0}} .
\end{aligned}
$$

Now, by (4.4),

$$
2 k(v, \Delta u)_{H^{0}}=2 k \int_{\partial \Omega} v \frac{\partial u}{\partial \nu} d s-2 k D(u, v)
$$

while

(4.10) $2 k(u, v)_{H^{1}}=2 k(u, v)_{H^{0}}+2 k D(u, v) \leqslant k\|u\|_{H^{1}}^{2}+k\|v\|_{H^{0}}^{2}+2 k D(u, v)$.

Hence, from (4.8), (4.9), (4.10),

$$
\|U\|_{H^{0}}^{2}-\left\|L_{k} U\right\|_{H^{0}}^{2} \leqslant k\|u\|_{H^{1}}^{2}+k\|v\|_{H^{0}}^{2}+\frac{1}{k^{2}} \int_{\partial \Omega} v^{2} d s+k^{4} \int_{\partial \Omega}\left|\frac{\partial u}{\partial \nu}\right|^{2} d s
$$

Remembering (4.1), the lemma follows from (4.11).

5. The Fully-Discrete Problem. For the approximate solution of (3.1) at each time step, we shall employ a finite-dimensional subspace $S_{h}^{q}$ of $H^{2}(\Omega) \times H^{1}(\Omega)$. This subspace will have the property that given any two-component vector $V \in \dot{H}^{2+s}$ $\times \dot{H}^{1+s} \subset H^{2} \times H^{1}$, there is a vector $\Psi$ in $S_{h}^{q}$ such that

$$
\|V-\Psi\|_{H^{l}} \leqslant C h^{s+1-l}\|V\|_{s+1}, \quad l=0,1,
$$

for all $0 \leqslant s \leqslant q$. Here, $C$ is a constant independent of $V$ and $h$. The construction of such spaces is discussed in [2], [3], [4], [8] and their references. Computational investigations of least squares procedures for elliptic boundary-value problems are reported in [7]. From (5.1), we deduce that

$$
\operatorname{Inf}_{\Phi \in S_{h}^{q}}\left\{\|\Phi-V\|_{H^{0}}+h\|\Phi-V\|_{H^{1}}\right\} \leqslant C h^{s+1}\|V\|_{s+1}, \quad s \leqslant q .
$$

At each time step in (3.1), one must approximate the solution of

$$
\begin{aligned}
L_{k} W=V & \text { in } \Omega, \\
w^{1}=0 & \text { on } \partial \Omega,
\end{aligned}
$$

where $w^{1}$ is the first component of $W$, and $V$ is obtained from the preceding time step. We now introduce the bilinear form 


$$
(\Phi, \Psi)_{\Lambda}=\left(L_{k} \Phi, L_{k} \Psi\right)_{H^{0}}+k^{4} \int_{\partial \Omega} \frac{\partial \psi_{1}}{\partial \nu} \frac{\partial \varphi_{1}}{\partial \nu} d s+\gamma_{k h} \int_{\partial \Omega} \psi_{2} \varphi_{2} d s
$$

where $\Psi=\left[\psi_{1}, \psi_{2}\right]^{T}$, and $\gamma_{k h} \geqslant 1 / k^{2}$ is a weight which will be chosen later. The above form is defined for all $\Phi, \Psi$ in $H^{2} \times H^{1}$, and $(,)_{\Lambda}$ defines an additional innerproduct on $H^{2} \times H^{1}$, i.e.,

$$
\|\Phi\|_{\Lambda}^{2}=(\Phi, \Phi)_{\Lambda}
$$

is a proper norm on $H^{2} \times H^{1}$. To see this, we note that $\|\Phi\|_{\Lambda}=0$ implies

$$
\begin{aligned}
\varphi_{1}-k \varphi_{2}=0 & \text { in } \Omega, \\
\varphi_{2}-k \Delta \varphi_{1}=0 & \text { in } \Omega, \\
\partial \varphi_{1} / \partial \nu=0 & \text { on } \partial \Omega .
\end{aligned}
$$

Eliminating $\varphi_{2}$ from (5.8), we get

$$
\varphi_{1}-k^{2} \Delta \varphi_{1}=0 \text { in } \Omega .
$$

(5.9), (5.10) imply $\varphi_{1}=0$. Hence, from (5.7), $\varphi_{2}=0$. Thus, $\|\Phi\|_{\Lambda}=0$ implies $\Phi=0$.

Lemma 5.1. Let $V \in \dot{H}^{2+s} \times \dot{H}^{1+s}$ and let $W$ be the corresponding solution of (5.3), (5.4). Then there is a unique element $\tilde{U}$ in $S_{h}^{q}$. minimizing $\|\Phi-W\|_{\Lambda}$ over all $\Phi$ in $S_{h}^{q}$. It is given by

$$
(\tilde{U}, F)_{\Lambda}=\left(V, L_{k} F\right)_{H^{0}}+k^{4} \int_{\partial \Omega} \frac{\partial w_{1}}{\partial \nu} \frac{\partial f_{1}}{\partial \nu} d s
$$

for all $F$ in $S_{h}^{q}$, where $w_{1}, f_{1}$ are the first components of $W$ and $F$ respectively.

Proof. By Pythagoras' theorem, the unique $\tilde{U}$ minimizing $\|\Phi-W\|_{\Lambda}^{2}$ satisfies

$$
(\tilde{U}, F)_{\Lambda}=(W, F)_{\Lambda} \text { for all } F \text { in } S_{h}^{q} .
$$

Now, if $V \in \dot{H}^{2+s} \times \dot{H}^{1+s}$, so is $W$, by Theorem 3.1. Hence, both components of $W$ vanish on $\partial \Omega$. Therefore,

$$
(W, F)_{\Lambda}=\left(V, L_{k} F\right)_{H^{0}}+k^{4} \int_{\partial \Omega} \frac{\partial w_{1}}{\partial \nu} \frac{\partial f_{1}}{\partial \nu} d s
$$

which proves the lemma.

We will now describe a family of fully-discrete schemes for solving (2.6). The schemes differ from one another only in the choice of the weight $\gamma_{k h}$. Let $p$ be a real number greater than or equal to 2 and let $\gamma_{k h}$ in (5.5) satisfy

$$
1 / k^{2} \leqslant \gamma_{k h} \leqslant C k^{2} h^{-p},
$$

where $C$ is a constant as $k, h \rightarrow 0$. Note that (5.14) implies that

$$
h \leqslant C k^{4 / p} \text { as } k, h \rightarrow 0 .
$$

For each choice of $p$, the algorithm is as follows. Given any initial data $W^{0}$ in $\dot{H}^{1+s} \times \dot{H}^{s}, s \geqslant 0$, we define a sequence of approximate solutions $\left\{\tilde{W}^{n}\right\}$ of (3.1) by means of 


$$
\begin{aligned}
\left(\tilde{W}^{n+1}, F\right)_{\Lambda} & =\left(\tilde{W}^{n}, L_{k} F\right)_{H^{0}}, \quad \text { for all } F \text { in } S_{h}^{q}, \\
\tilde{W}^{0} & =W^{0},
\end{aligned}
$$

$\tilde{W}^{n+1}$ being sought in $S_{h}^{q}$. Thus, given a basis for $S_{h}^{q}$, finding $\tilde{W}^{n+1}$ from $\tilde{W}^{n}$ necessitates solving a system of linear equations. This system always has a unique solution because $S_{h}^{q}$ is finite dimensional and \|\|$_{\Lambda}$ is a norm on $S_{h}^{q}$. It should be noted that the scheme is defined for any initial data in $H_{0}^{1}(\Omega) \times L^{2}(\Omega)$. The above scheme is conceptually different from that described in [4] for the heat equation. For instance, finding $\tilde{W}^{1}$ from $W^{0}$ by means of $(5.16)$, is not the same as minimizing $\|\Phi-W\|_{\Lambda}^{2}$ over $S_{h}^{q}$ where $W$ is the exact solution of $L_{k} W=W^{0}$. Indeed, according to (5.11) in Lemma 5.1, the unique element $\widetilde{U}^{1}$ in $S_{h}^{q}$, which minimizes $\|\Phi-W\|_{\Lambda}^{2}$, satisfies a different linear system, the right-hand side of which involves $\partial w_{1} / \partial \nu$. In (5.16), we have effectively replaced this unknown normal derivative by zero. The scheme (5.16), (5.17) is a combination of least squares and penalty methods. At this point, we wish to make the following observation. Given $V$ in $\dot{H}^{2+s} \times \dot{H}^{1+s}$, let $\widetilde{U}$ be defined by (5.11), and let $\widetilde{W}$ be defined by

$$
(\tilde{W}, F)_{\Lambda}=\left(V, L_{k} F\right)_{H^{0}} \text { for all } F \text { in } S_{h}^{q} .
$$

Then,

$$
(\tilde{U}-\tilde{W}, F)_{\Lambda}=k^{4} \int_{\partial \Omega} \frac{\partial w_{1}}{\partial \nu} \frac{\partial f_{1}}{\partial \nu} d s \text { for all } F \text { in } S_{h}^{q}
$$

Hence,

$$
\|\tilde{U}-\tilde{W}\|_{\Lambda}^{2} \leqslant k^{4}\left\{\int_{\partial \Omega}\left|\frac{\partial w_{1}}{\partial \nu}\right|^{2} d s\right\}^{1 / 2}\left\{\int_{\partial \Omega}\left|\frac{\partial}{\partial \nu}\left(\tilde{u}_{1}-\tilde{w}_{1}\right)\right|^{2} d s\right\}^{1 / 2} .
$$

From (5.5), we see that

$$
k^{2}\left\{\int_{\partial \Omega}\left|\frac{\partial}{\partial \nu}\left(\tilde{u}_{1}-\tilde{w}_{1}\right)\right|^{2} d s\right\}^{1 / 2} \leqslant\|\tilde{U}-\tilde{W}\|_{\Lambda} .
$$

Hence,

$$
\|\tilde{U}-\tilde{W}\|_{\Lambda} \leqslant k^{2}\left\{\int_{\partial \Omega}\left|\frac{\partial w_{1}}{\partial \nu}\right|^{2} d s\right\}^{1 / 2} .
$$

Next, by Theorem 3.1, if $V$ is in $\dot{H}^{2+s} \times \dot{H}^{1+s}$, so is $W$, the solution of (5.3), (5.4), and

$$
\|W\|_{1} \leqslant\|V\|_{1} .
$$

Since

$$
\int_{\partial \Omega}\left|\frac{\partial w_{1}}{\partial \nu}\right|^{2} d s \leqslant C\left\|w_{1}\right\|_{H^{2}}^{2}
$$

we get, from (5.22), (5.23),

$$
\|\tilde{U}-\tilde{W}\|_{\Lambda} \leqslant C k^{2}\|V\|_{1} .
$$

The idea behind the scheme (5.16), (5.17) lies in this last inequality, which shows 
that using the wrong value for the normal derivative in (5.16) leads to an error of order $k^{2}$. As will be seen below in Lemma 5.4, this means that the scheme (5.16), (5.17) is still consistent with the analytic problem (2.6).

With $\tilde{W}^{n}$ as in (5.16), put $\tilde{W}^{1}=E_{k h} W^{0}, \tilde{W}^{n}=E_{k h} \tilde{W}^{n-1}=E_{k h}^{n} W^{0}$. The family of discrete solution operators $\left\{E_{k h}^{n}\right\}$ is bounded uniformly in the $H^{1} \times H^{0}$ norm as $k, h \rightarrow 0, n \rightarrow \infty, n k \leqslant T$. Thus, the fully-discrete scheme (5.16) is unconditionally stable. This is the content of $h$,

LEMMA 5.2.For all $W^{0}$ in $H_{0}^{1} \times H^{0}$ and all $0 \leqslant n k \leqslant T$, we have, independently of

$$
\left\|E_{k h}^{n} W^{0}\right\|_{H^{0}} \leqslant C e^{T / 2}\left\|W^{0}\right\|_{H^{0}} .
$$

Proof. By (5.16) and Schwarz's inequality,

$$
\left\|\tilde{W}^{n+1}\right\|_{\Lambda}^{2} \leqslant\left\|\tilde{W}^{n}\right\|_{H^{0}}\left\|L_{k} \tilde{W}^{n+1}\right\|_{H^{0}} \leqslant\left\|\tilde{W}^{n}\right\|_{H^{0}}\left\|\tilde{W}^{n+1}\right\|_{\Lambda} .
$$

Hence, $\left\|E_{k h}^{n+1} W^{0}\right\|_{\Lambda} \leqslant\left\|E_{k h}^{n} W^{0}\right\|_{H^{0}}$.

By Lemma 4.2, the definition of the $\Lambda$ norm, and the fact that $\gamma_{k h} \geqslant 1 / k^{2}$,

$$
\left\|E_{k h}^{n} W^{0}\right\|_{H^{0}}^{2} \leqslant \frac{1}{1-k}\left\|E_{k h}^{n} W^{0}\right\|_{\Lambda}^{2} \leqslant \frac{1}{1-k}\left\|E_{k h}^{n-1} W^{0}\right\|_{H^{0}}^{2} .
$$

The lemma follows from (5.28).

LemmA 5.3. Let $0 \leqslant s \leqslant q$, and let $W \in \dot{H}^{2+s} \times \dot{H}^{1+s}$. Fix $p \geqslant 2$ in (5.14). Then there is a constant $C$ independent of $W$ and $h$ such that

$$
\operatorname{Inf}_{\Phi \in S_{h}^{q}}\|\Phi-W\|_{\Lambda} \leqslant C k h^{(2 s+1)-p / 2}\|W\|_{1+s} .
$$

Proof. For any $U=\left[u_{1}, u_{2}\right]^{T} \in H^{2} \times H^{1}$, we have

$$
\|U\|_{\Lambda}^{2}=\left\|u_{1}\right\|_{H^{1}}^{2}-2 k\left(u_{1}, u_{2}\right)_{H^{1}}-2 k\left(u_{2}, \Delta u_{1}\right)_{H^{0}}+\left\|u_{2}\right\|_{H^{0}}^{2}+k^{2}\left\|u_{2}\right\|_{H^{1}}^{2}+k^{2}\left\|\Delta u_{1}\right\|_{H^{0}}^{2}
$$

$$
\begin{aligned}
& +\gamma_{k h} \int_{\partial \Omega}\left|u_{2}\right|^{2} d s+k^{4} \int_{\partial \Omega}\left|\frac{\partial u_{1}}{\partial \nu}\right|^{2} d s \\
\leqslant & C\left(\left\|u_{1}\right\|_{H^{1}}^{2}+\left\|u_{2}\right\|_{H^{0}}^{2}\right)+C k^{2}\left(\left\|u_{1}\right\|_{H^{2}}^{2}+\left\|u_{2}\right\|_{H^{1}}^{2}\right)+\gamma_{k h} \int_{\partial \Omega}\left|u_{2}\right|^{2} d s
\end{aligned}
$$

where we have used (5.24). Hence,

$$
\begin{aligned}
\|U\|_{\Lambda}^{2} & \leqslant C k^{2}\left\{\frac{1}{k^{2}}\left\|u_{1}\right\|_{H^{1}}^{2}+\frac{1}{k^{2}}\left\|u_{2}\right\|_{H^{0}}^{2}+\frac{\gamma_{k h}}{k^{2}} \int_{\partial \Omega}\left|u_{2}\right|^{2} d s+\left\|u_{1}\right\|_{H^{2}}^{2}+\left\|u_{2}\right\|_{H^{1}}^{2}\right\} \\
& \leqslant C k^{2}\left\{k^{2} h^{-p}\|U\|_{H^{0}}^{2}+\|U\|_{H^{1}}^{2}+h^{-p} \int_{\partial \Omega}\left|u_{2}\right|^{2} d s\right\}
\end{aligned}
$$

where we have used (5.14), (5.15). To estimate the boundary integral in the last inequality, we use Lemma 4.1 with $\epsilon=h$. We obtain

$$
\begin{aligned}
\|U\|_{\Lambda}^{2} & \leqslant C k^{2}\left\{k^{2} h^{-p}\|U\|_{H^{0}}^{2}+h^{-(p+1)}\|U\|_{H^{0}}^{2}+\|U\|_{H^{1}}^{2}+h^{-(p-1)}\|U\|_{H^{1}}^{2}\right\} \\
& \leqslant C k^{2} h^{-(p+1)}\left\{\|U\|_{H^{0}}^{2}+h^{2}\|U\|_{H^{1}}^{2}\right\}
\end{aligned}
$$

so that

$$
\|U\|_{\Lambda} \leqslant C k h^{-(p+1) / 2}\left\{\|U\|_{H^{0}}+h\|U\|_{H^{1}}\right\} .
$$


Consequently, using (5.33) and (5.2),

$$
\operatorname{Inf}_{\Phi \in S_{h}^{q}}\|\Phi-W\|_{\Lambda} \leqslant C k h^{(2 s+1-p) / 2}\|W\|_{1+s}
$$

as required.

Recall that $E(t)$ is the solution operator for the analytic problem $(2.6), E_{k}(t)$ that for the semi-discrete problem (3.1), (3.2), and $E_{k h}(t)=E_{k h}^{n}$ is the fully-discrete solution operator. In Lemma 5.2, the stability of the fully-discrete scheme was proved. The next lemma shows that the scheme is consistent with (2.6) whenever $q$ in $(5.1)$ is chosen greater than $(p-1) / 2$.

Lemma 5.4. Fix $a p \geqslant 2$, in (5.14) and let $V \in \dot{H}^{q+2} \times \dot{H}^{q+1}$. Then

$$
\left\|E_{k h} V-E_{k}(k) V\right\|_{H^{0}} \leqslant C k\left(h^{(2 q+1-p) / 2}\|V\|_{q+1}+k\|V\|_{1}\right) .
$$

Proof. By Lemma 4.2 and $\gamma_{k h} \geqslant 1 / k^{2}$,

$$
\left\|E_{k h} V-E_{k}(k) V\right\|_{H^{0}} \leqslant \sqrt{\frac{1}{1-k}}\left\|E_{k h} V-E_{k}(k) V\right\|_{\Lambda} .
$$

Since $V \in \dot{H}^{2+s} \times \dot{H}^{1+s}, s \geqslant 0, \tilde{U}$ defined by (5.11) minimizes $\left\|\Phi-E_{k}(k) V\right\|_{\Lambda}$ over $S_{h}^{q}$. Hence, using Lemma 5.3 and Theorem 3.1,

$$
\left\|\tilde{U}-E_{k}(k) V\right\|_{\Lambda} \leqslant C k h^{(2 q+1-p) / 2}\left\|E_{k}(k) V\right\|_{q+1} \leqslant C k h^{(2 q+1-p) / 2}\|V\|_{q+1} .
$$

Next, $\tilde{W}=E_{k h} V$ defined by (5.18) satisfies (5.25). Hence, by the triangle inequality and (5.36),

$$
\left\|E_{k h} V-E_{k}(k) V\right\|_{H^{0}} \leqslant C k\left(h^{(2 q+1-p) / 2}\|V\|_{q+1}+k\|V\|_{1}\right)
$$

as required.

Using the Lax Equivalence Theorem, it follows from Lemmas 5.2 and 5.4 that the scheme (5.16), (5.17) converges to the exact solution of the analytic problem (2.6) as $k, h \rightarrow 0 ; n k \leqslant T$, for all initial data $W^{0}$ in $H_{0}^{1}(\Omega) \times L^{2}(\Omega)$. For such data, the theory does not provide any information on the rate of convergence. On the other hand, for smooth $W^{0}$, we have the following

THEOREM 5.1. Let $\sigma=\operatorname{Max}(q+1,2)$ and let $W^{0} \in \dot{H}^{\sigma+1} \times \dot{H}^{\sigma}$; then there is a constant, $C_{T}$, depending only on $T$, such that, for $0 \leqslant t=n k \leqslant T$,

$$
\left\|E_{k h}(t) W^{0}-E(t) W^{0}\right\|_{H^{0}} \leqslant C_{T}\left\{h^{(2 q+1-p) / 2}\left\|W^{0}\right\|_{q+1}+k\left\|W^{0}\right\|_{2}\right\} .
$$

Proof. We use the identity

$$
\begin{aligned}
E_{k h}(t) W^{0}-E_{k}(t) W^{0} & =E_{k h}^{n} W^{0}-E_{k}(n k) W^{0} \\
& =\sum_{j=0}^{n-1} E_{k h}^{n-1-j}\left(E_{k h}-E_{k}(k)\right) E_{k}(j k) W^{0}
\end{aligned}
$$

and Lemma 5.2 to obtain

$$
\left\|E_{k h}(t) W^{0}-E_{k}(t) W^{0}\right\|_{H^{0}} \leqslant C \frac{T}{k} e^{T / 2} \operatorname{Max}_{j}\left\|\left(E_{k h}-E_{k}(k)\right) E_{k}(j k) W^{0}\right\|_{H^{0}} .
$$


Using Lemma 5.4 and Theorem 3.1,

$$
\begin{aligned}
& \left\|\left(E_{k h}-E_{k}(k)\right) E_{k}(j k) W^{0}\right\|_{H^{0}} \\
& \quad \leqslant C k\left\{k\left\|E_{k}(j k) W^{0}\right\|_{1}+h^{(2 q+1-p) / 2}\left\|E_{k}(j k) W^{0}\right\|_{q+1}\right\} \\
& \quad \leqslant C k\left\{h^{(2 q+1-p) / 2}\left\|W^{0}\right\|_{q+1}+k\left\|W^{0}\right\|_{1}\right\} .
\end{aligned}
$$

Hence,

$$
\left\|E_{k h}(t) W^{0}-E_{k}(t) W^{0}\right\|_{H^{0}} \leqslant C T e^{T / 2}\left\{h^{(2 q+1-p) / 2}\left\|W^{0}\right\|_{q+1}+k\left\|W^{0}\right\|_{1}\right\} .
$$

Next, by Theorem 3.2,

$$
\left\|E(t) W^{0}-E_{k}(t) W^{0}\right\|_{H^{0}} \leqslant C_{T} k\left\|W^{0}\right\|_{2} .
$$

The result follows from (5.43) and (5.44).

Remarks. As a consequence of (5.15), the error bound (5.39) has the form

$$
\left\|E_{k h}(t) W^{0}-E(t) W^{0}\right\|_{H^{0}} \leqslant C_{T}\left\{k\left\|W^{0}\right\|_{2}+k^{(4 q+2-2 p) / p}\left\|W^{0}\right\|_{1+q}\right\} .
$$

Hence, the smallest possible error is $O(k)$ and it occurs when $q$ and $p$ are such that

$$
p=(4 q+2) / 3 \text {. }
$$

Let the initial data $W^{0}$ be sufficiently smooth. Then, given any positive integer value of $q$ in (5.1), we obtain $O(k)$ accuracy by choosing $p$ to satisfy (5.46). If we choose $q=1$ and take for $S_{h}^{q}$, for example, the space of two component vectors where the first component is a quadratic spline and the second component is piecewise linear, we obtain $O(k)$ accuracy in the $H_{0}^{1} \times L^{2}$ norm, by choosing $p=2$ in (5.14). However, this requires

$$
h \leqslant C k^{2} \text { as } k, h \rightarrow 0 .
$$

Such a constraint on $h$ is not practical. By using higher values of $q$, i.e., smoother trial functions, we can obtain $O(k)$ accuracy with a more favorable mesh inequality. For example, using quintic and quartic splines, i.e., $q=4$, we have $O(k)$ accuracy, with $h \leqslant C k^{2 / 3}$. Other choices of $p$ and $q$ yielding $O(k)$ accuracy are summarized in Table I below.

The above remarks concerning $O(k)$ accuracy assume that $W^{0} \in \dot{H}^{1+\sigma} \times \dot{H}^{\sigma}$ with $\sigma=\operatorname{Max}(q+1,2)$. More generally, we have

TheOrem 5.2. Let $W^{0} \in \dot{H}^{s+1} \times \dot{H}^{s}, s \geqslant 0$. Choose a positive integer $q$ in (5.1) and let $p$ in (5.14) satisfy (5.46). Then, for $0 \leqslant t=n k \leqslant T$,

$$
\left\|E_{k h}(t) W^{0}-E(t) W^{0}\right\|_{H^{0}} \leqslant C_{T} k^{\operatorname{Min}(1, s /(1+q))}\left\|W^{0}\right\|_{s} .
$$

Proof. For arbitrary $W^{0} \in H_{0}^{1} \times L^{2}$, we have from Theorem 2.1, Lemma 5.2 and the triangle inequality,

$$
\left\|E(t) W^{0}-E_{k h}(t) W^{0}\right\|_{H^{0}} \leqslant C_{T}\left\|W^{0}\right\|_{H^{0}} .
$$

If $W^{0} \in \dot{H}^{q+2} \times \dot{H}^{1+q}$, we have, from (5.45) and (5.46),

$$
\left\|E(t) W^{0}-E_{k h}(t) W^{0}\right\|_{H^{0}} \leqslant C_{T} k\left\|W^{0}\right\|_{1+q} .
$$


TABLE I

\begin{tabular}{crrl}
\hline $\begin{array}{l}\text { Degree of } \\
\text { Splines in } S_{h}^{q}\end{array}$ & $q$ & $p$ & Mesh Inequality \\
\hline $2 \times 1$ & 1 & 2 & $h \leqslant C k^{2}$ \\
$3 \times 2$ & 2 & $10 / 3$ & $h \leqslant C k^{6 / 5}$ \\
$4 \times 3$ & 3 & $14 / 3$ & $h \leqslant C k^{6 / 7}$ \\
$5 \times 4$ & 4 & 6 & $h \leqslant C k^{2 / 3}$ \\
$6 \times 5$ & 5 & $22 / 3$ & $h \leqslant C k^{6 / 11}$ \\
$7 \times 6$ & 6 & $26 / 3$ & $h \leqslant C k^{6 / 13}$ \\
$8 \times 7$ & 7 & 10 & $h \leqslant C k^{2 / 5}$ \\
$9 \times 8$ & 8 & $34 / 3$ & $h \leqslant C k^{6 / 17}$ \\
$10 \times 9$ & 9 & $38 / 3$ & $h \leqslant C k^{6 / 19}$ \\
$11 \times 10$ & 10 & 14 & $h \leqslant C k^{2 / 7}$ \\
\hline
\end{tabular}

Next, the spaces $\dot{H}^{s+1} \times \dot{H}^{s}$ with the norm (2.7) have the interpolation property discussed in [4,Lemma 2.2]. Hence, the result follows from (5.49), (5.50).

Department of Mathematics

University of New Mexico

Albuquerque, New Mexico 87131

1. S. Agmon, Lectures on Elliptic Boundary Value Problems, Van Nostrand Math. Studies, no. 2, Van Nostrand, New York, 1965. MR 31 \#2504.

2. G. Birkhoff, M. Schultz \& R. VARGA, "Piecewise Hermite interpolation in one and two variables with applications to partial differential equations," Numer. Math., v. 11, 1968, pp. 232-256. MR 37 \#2404,

3. J. H. Bramble \& S. Hilbert, "Estimation of linear functionals on Sobolev spaces with application to Fourier transforms and spline interpolation," SIAM J. Numer. Anal., v. 7, 1970, pp. 112-124. MR 41 \#7819.

4. J. H. Bramble \& V. Thomée, "Semi-discrete least squares methods for a parabolic boundary value problem," Math. Comp. v. 26, 1972, pp. 633-648.

5. T. Kato, Perturbation Theory for Linear Operators, Die Grundlehren der math. Wissenschaften, Band 132, Springer-Verlag, New York, 1966. MR 34 \#3324.

6. J. L. Lions \& E. Magenes, Problèmes aux limites homogènes et applications. Vol. 1, Travaux et Recherches Mathématiques, no. 17, Dunod, Paris, 1968. MR 40 \#12.

7. S. M. SERbin, Doctoral Dissertation, Department of Mathematics, Cornell University, Ithaca, N.Y., 1971.

8. R. S. VARGA, Functional Analysis and Approximation Theory in Numerical Analysis, Regional Conference Series in Appl. Math., vol. 3, SIAM, Philadelphia, Pa., 1971. 